Harald Freiling

\title{
Kostengünstige Vertretungsverträge statt berufsqualifizierender Seiteneinstiege - eine Problemskizze am Beispiel des Landes Hessen
}

\section{Zusammenfassung}

Dass mit steigendem Lehrkräftemangel immer mehr Seiteneinsteiger ${ }^{\star}$ innen eingestellt werden, ist in den letzten Jahren zunehmend thematisiert und kritisiert worden. Sie verfügen in der Regel nicht über schulpädagogische Qualifikationen, werden aber dennoch mit hoher Stundenzahl im Unterricht eingesetzt. Allerdings wird ihnen eine berufsbegleitende Qualifizierung geboten, mit der sie eine pädagogische Fachausbildung bis hin zur Gleichstellung mit einem Zweiten Staatsexamen nachholen sollen (vgl. den Beitrag von Driesner \& Arndt in diesem Heft). Die kritische Auseinandersetzung mit der Arbeitssituation dieser Gruppe übersieht aber nur zu leicht, dass es daneben eine ebenfalls große Gruppe von unausgebildeten „Vertretungskräften“ gibt, die in der Schule unter noch schlechteren Bedingungen und ohne jede Qualifikations- und berufliche Lebensperspektive unterrichten. Das Ausmaß und die Bedingungen der Beschäftigung dieser Gruppe und deren Auswirkungen auf den Unterricht sind Gegenstand des folgenden Beitrags, der sich auf das Bundesland Hessen konzentriert. Es gibt diese „Vertretungslehrkräfte“ aber auch in anderen Bundesländern, beispielsweise in Berlin, wo sie als "Lehrkräfte ohne volle Lehrbefähigung“ (LovL) bezeichnet werden. Da diese Vertretungskräfte in der Statistik der Kultusministerkonferenz nicht erfasst werden, gibt es jedoch keinen Überblick über ihre bundesweite Verbreitung. Deshalb behandeln wir ihre Problemlage zunächst im Rahmen einer länderspezifischen Fallstudie.

Schlüsselwörter: Lehrkräftemangel, Hessen, Vertretungsverträge, prekäre Beschäftigung, Quereinstieg, Gleichstellung (mit einem Lehramt), Unterrichtsqualität 


\title{
Low-Cost Agency Agreements instead of Certificated Career Changes - an Outline of the Problem, Using the Example of Hesse
}

\begin{abstract}
It has been increasingly discussed and criticized in recent years that due to the growing teacher shortage, more and more career changers are employed. They usually have no school-specific pedagogical qualifications, but they have a high teaching load. However, an in-service training is offered to them; thereby, they are supposed to catch up on teacher training up to the Second State Examination (cp. the contribution by Driesner \& Arndt in this issue). The critical discussion of this group's working situation ignores very easily that there is also another big group of untrained "supply teachers", who teach at school under even worse circumstances, without any qualification or vocational perspective. This contribution deals with the extent and the conditions of their employment and resulting effects on the quality of teaching, focusing on Hesse. But these "supply teachers" also work in other German Länder, for example in Berlin, where they are called "teachers without full teaching authorization". As these supply teachers are not recorded in the statistics of the Standing Conference of the Ministers of Education and Cultural Affairs of the Länder in the Federal Republic of Germany, there are no data regarding their nationwide distribution. Therefore, for the time being, we use a specific case-study to illustrate their complex of problems.

Keywords: teacher shortage, Hesse, agency agreements, precarious occupation, career change, equal treatment (to fully qualified teachers), quality of teaching

Die Bilanz der Kultusministerkonferenz (KMK) zur Neueinstellung von Lehrkräften im Jahr 2018 machte Schlagzeilen, denn sie wies einen neuen Höchstwert bei der Zahl der Einstellung von Seiteneinsteiger*innen aus, die nicht „über die erste Lehramtsprüfung verfügen und ohne das Absolvieren des eigentlichen Vorbereitungsdienstes in den Schuldienst eingestellt werden“" (KMK, 2019, S. 32). Besonders viele Seiteneinsteiger*innen wurden in Sachsen (1.174), Berlin (1.057), NordrheinWestfalen (1.006) und Niedersachsen (478) eingestellt; in Sachsen lag ihr Anteil an allen Einstellungen bei 50,2 Prozent, in Berlin bei 40,1 Prozent. Besonders niedrige Zahlen meldeten Bayern (0), Rheinland-Pfalz (10) und Hessen (44, davon 43 an beruflichen Schulen). 2019 ging die Gesamtzahl der mit Seiteinsteiger*innen besetzten Stellen gegenüber 2018 von 4.786 auf 3.265 zurück (KMK, 2020). Hessen liegt mit 34 Einstellungen (davon 10 in beruflichen Fächern) auch 2019 weiter am unteren Ende. Der folgende Beitrag versucht, diese Diskrepanz zu erklären, und zeigt die Lücken in der Erfassung von Lehrkräften ohne Lehramt durch die KMK auf.
\end{abstract}




\section{Die Einstellungsbilanz der KMK}

Tillmann erklärt den sehr niedrigen Anteil von Seiteneinsteiger*innen in Bayern, Hessen oder im Saarland, die noch 2017 der KMK keine einzige Einstellung einer Lehrkraft ohne Lehramt gemeldet hatten, unter anderem mit „unterschiedlichen Anstrengungen bei der Ausbildung von Lehrkräften“ sowie einer „unterschiedliche[n] Attraktivität der Länder als Arbeitgeber“ (Tillmann, 2019, S. 13). Beide Vermutungen lassen sich für Hessen ausschließen, da das Bundesland seit Jahren unter Bedarf ausbildet, sich bei der Einstellung in hohem Maß aus anderen Bundesländern bedient und mindestens bei der Pflichtstundenzahl der Lehrkräfte und ihrer Bezahlung mit Nullrunden bei der Beamtenbesoldung in den Jahren 2015 und 2016 kaum als besonders attraktiver Arbeitgeber gelten kann.

Der Schlüssel für die Erklärung der niedrigen Zahl von Seiteneinsteigerinnen ist ausschließlich in der unterschiedlichen Definition des Begriffs durch die Kultusministerien der Länder zu suchen. Nach der Definition der KMK sind dies Lehrkräfte, „die in der Regel über einen Hochschulabschluss, nicht jedoch über die erste Lehramtsprüfung verfügen und ohne das Absolvieren des eigentlichen Vorbereitungsdienstes in den Schuldienst eingestellt werden“ und die „über ihre fachlichen Kenntnisse hinaus eine pädagogische Zusatzqualifikation“ erhalten, die „teilweise auch berufsbegleitend vermittelt wird“ (KMK, 2019, S. 32). Damit ist davon auszugehen, dass die Bundesländer auch nur die Zahl der Seiteneinsteiger*innen melden, die im Rahmen eines landesspezifischen Qualifizierungsangebots eingestellt werden. Da Hessen über solche Qualifizierungsprogramme nicht verfügt oder diese - wie unten dargestellt - nicht anwendet, meldet es auch keine Zahlen oder nur ganz wenige Fälle, insbesondere im Bereich der beruflichen Schulen.

Dass dies von anderen Bundesländern anders gehandhabt wird, zeigt ein Vergleich mit dem Bundesland Berlin: Eine Auswertung interner Zahlen für den Gesamtpersonalrat macht deutlich, dass nicht nur die Personen in den strukturierten Seiteneinstiegsprogrammen wie dem viersemestrigen Q-Master, sondern auch die neu eingestellten „Lehrkräfte ohne volle Lehrbefähigung“ (LovL) als Seiteneinsteiger*innen ganz oder teilweise an die KMK gemeldet werden. Bei den LovL handelt es sich um Personen, die die Einstiegsvoraussetzungen für den mit einer Qualifizierung verbundenen Seiteneinstieg (u.a. Examen in einem Mangelfach, Studien in einem zweiten Unterrichtsfach) nicht erfüllen. Aufgrund des Lehrkräftemangels werden sie trotzdem eingestellt, und zwar ohne jede Qualifikationsmaßnahme mit einem in der Regel auf zwei Jahre befristeten Arbeitsvertrag.

Dass Hessen so gut wie keine Seiteneinsteiger*innen meldet, heißt aber nicht, dass es in hessischen Schulen keine Lehrkräfte gibt, die ohne einen Lehramtsabschluss unterrichten. Dies werden wir im Folgenden ausführlich darstellen. Dabei wer- 
den ausschließlich die allgemeinbildenden Schulen in den Blick genommen, da der Lehrkräfteeinsatz an beruflichen Schulen mit den Lehrkräften für Fachpraxis und vielen Quereinsteiger*innen aus der beruflichen Praxis, die nicht über ein Lehramtsstudium verfügen, eigenen Traditionen und Gesetzmäßigkeiten folgt.

\section{Unterrichtsabdeckung durch Vertretungskräfte}

Tatsächlich ist die Zahl der Lehrkräfte, die nicht über eine grundständige Lehrkräfteausbildung mit einem Ersten und Zweiten Staatsexamen verfügen und trotzdem in allen Bereichen des Pflichtunterrichts mit allen damit verbundenen Aufgaben eingesetzt werden, hoch und ansteigend. Auch in Hessen wird der Lehrkräftemangel vor allem durch Lehrkräfte ohne Lehramtsabschluss aufgefangen. Diese werden - anders als in anderen Bundesländern - jedoch nicht mit dem Ziel einer berufsbegleitenden Professionalisierung und der Perspektive einer dauerhaften Beschäftigung im Lehrberuf eingestellt, sondern ohne vertiefte Prüfung der vorhandenen Qualifikationen als Vertretungslehrer*innen befristet beschäftigt, um so einen konkreten Vertretungsbedarf zu decken. Angesichts des wachsenden Mangels an ausgebildeten Lehrkräften wurde aus den ursprünglichen „echten Vertretungen“ für eine Lehrkraft in Elternzeit oder in einer Familienpause ein Dauerzustand zur Abdeckung des Regelunterrichts, bei dem sich die befristet Beschäftigten von Jahr zu Jahr von einem Vertrag zum anderen hangeln müssen.

Rechtsgrundlagen für die Einstellung dieser Vertretungskräfte, die einen wachsenden Teil des Pflichtunterrichts abdecken, sind $\$ 62$ des Hessischen Lehrerbildungsgesetzes (HLbG) und $\$ 83$ der Durchführungsverordnung zu demselben Gesetz (HLbGDV). Unterrichts- und Erziehungsaufgaben in öffentlichen Schulen dürfen danach mit einer „Unterrichtserlaubnis“ übernommen werden, die von den Staatlichen Schulämtern erteilt wird. Eine solche Unterrichtserlaubnis kann auch ohne den Nachweis einer fachlichen Qualifikation oder ohne eine Überprüfung der unterrichtlichen Fähigkeiten erteilt werden, „wenn ein unabweisbares Unterrichtsbedürfnis vorliegt und nachhaltige Bemühungen zur Gewinnung einer ausgebildeten Lehrkraft ohne Ergebnis geblieben sind“ ( $\$ 83$ HLbG-DV). Auf der Grundlage einer solchen Unterrichtserlaubnis wurden in Hessen viele tausend Lehrkräfte eingestellt, ohne dass sie als Seiteneinsteiger*innen im Sinn der KMK erfasst werden. Darunter sind Lehramtsstudent*innen, Personen mit und ohne Hochabschluss und mit sehr unterschiedlichen Berufserfahrungen, ohne dass damit eine Aussage über ihre konkrete Arbeit im Unterricht verbunden ist. Sie erhalten keine Aus- und Fortbildung; die Anleitung erfolgt ausschließlich im Rahmen des kollegialen Miteinanders durch Lehrkräfte des Stammkollegiums. ${ }^{1}$

1 Im Schuljahr 2019/2020 entwickelte die Hessische Lehrkräfteakademie eine erste „Unterstützungsmaßnahme für TVH-Kräfte ohne Lehramt an Grundschulen“ mit begrenzten Einzelveranstaltungen zu Fragen des Schulrechts, der Unterrichtsmethodik und zur Rolle der 


\section{Prekäre Beschäftigung ohne Qualifizierung}

Die Beschäftigung erfolgt im Rahmen eines befristeten Arbeitsvertrags auf der Grundlage des Tarifvertrags Hessen (TVH) und der Befristungsmöglichkeiten nach $\$ 14$ des Teilzeit- und Befristungsgesetzes. Insbesondere durch die Verschiebung des Einstellungsbedarfs von der zulässigen „Vertretung eines anderen Arbeitnehmers“ ( $\$ 14$ Abs. 1 Punkt 3 TzBfG) zu der arbeitsrechtlich unzulässigen Abdeckung eines dauerhaft bestehenden Bedarfs und aufgrund der veränderten Rechtsprechung des Bundesarbeitsgerichts konnten zahlreiche Personen in den letzten Jahren eine Entfristung ihres Arbeitsverhältnisses durchsetzen. Diese Entfristung ändert jedoch nichts an der jeweiligen Entgeltgruppe des TVH; Grundlage der Eingruppierung ist die jeweilige Schulform und die formale Qualifikation. Konkret bedeutet dies, dass es in Hessen Lehrkräfte gibt, die aufgrund eines fehlenden Hochschulabschlusses an einer Grundschule in der Entgeltgruppe 6 seit Jahren alle Aufgaben einer Lehrkraft im Unterricht, als Klassenlehrkraft und in allen außerunterrichtlichen Bereichen wahrnehmen. Beschäftigte an einem Gymnasium mit abgeschlossener wissenschaftlicher Hochschulbildung, die aufgrund ihres Studiums die Fähigkeit zum Unterrichten in mindestens zwei Fächern haben, werden zwar mit einer Vergütung nach Entgeltgruppe 13 den Lehrkräften mit Lehramt gleichgestellt, aber auch sie haben als Vertretungslehrkräfte keine Perspektive auf eine Entfristung ihres Arbeitsvertrags oder eine Verbeamtung durch die Gleichstellung mit einem Lehramt.

\section{Das Kultusministerium verweigert die Auskunft}

Das Hessische Kultusministerium (HKM) hat auch in mehreren parlamentarischen Anfragen keine Auskunft über die tatsächliche Qualifikation und den Anteil der Lehrkräfte ohne Lehramt gegeben. Erst eine breite öffentliche Debatte über den Unterricht, der von nicht grundständig ausgebildeten Lehrkräften erteilt wird, führte im Januar $2020 \mathrm{zu}$ einer Aussage des HKM, wonach rund 4.900 der rund 60.000 hessischen Lehrkräfte über keine Lehramtsbefähigung verfügen und somit als Quereinsteiger ${ }^{\star}$ innen zu gelten hätten. Ausgelöst wurde die Debatte durch ein Dossier des Hessischen Rundfunks (hr), der das Thema im Rahmen der ARDThemenwoche „Bildung“ aufgegriffen hatte.

Die Zahl der Lehrkräfte in Hessen, die keinen Lehramtsabschluss haben, ist lediglich auf dem Umweg über die Entwicklung der Zahl der Lehrkräfte in einem befristeten Arbeitsverhältnis zu erfassen. In einer internen Aufstellung des Hessischen Innenministeriums wurde die Zahl der befristet beschäftigten Lehrkräfte an allen Schulen am 01.01.2019 mit 5.761 angegeben, die Zahl der so besetzten Stellen mit

Lehrkraft. Die Teilnahme an den Fortbildungen ist für die Fragen der tariflichen Eingruppierung und der Perspektiven im Beruf ohne Bedeutung. 
3.377. Der Anteil an den Lehrpersonen betrug danach 9,7 Prozent, an den Stellen 6,6 Prozent. Den engen Zusammenhang mit dem wachsenden Lehrkräftemangel, der nicht mehr durch ausgebildete Lehrkräfte behoben werden kann, dokumentiert auch die Zahl der befristet beschäftigten Lehrkräfte an Grundschulen, die von $1.278 \mathrm{im}$ Jahr 2016 auf 1.525 im Jahr 2017 und 1.633 im Jahr 2018 anstieg. ${ }^{2}$ Dabei kann insbesondere für die Grundschulen die Gleichsetzung der Zahl der befristet Beschäftigten mit der Zahl nicht ausgebildeter Lehrkräfte nicht angezweifelt werden, weil anders als noch vor zehn Jahren jede ausgebildete Grundschullehrkraft unmittelbar nach dem Referendariat in einem unbefristeten Arbeits- oder Beamtenverhältnis eingestellt wird.

\section{Vertretungskräfte vorrangig in sozialen Brennpunkten}

Die Verweigerung differenzierter Zahlen erschwert auch die Analyse regionaler Zusammenhänge.

Die letzten Zahlen, die Auskunft geben, wie viele Lehrkräfte in den 15 Staatlichen Schulämtern und in den einzelnen Schulformen nicht über einen Lehramtsabschluss verfügen, stammen aus dem Februar 2017. An den folgenden Befunden dürfte sich wenig geändert haben:

- Besonders hoch war der Anteil der Lehrkräfte ohne Lehramtsabschluss an den Grundschulen und den Integrierten Gesamtschulen; besonders niedrig ist der Anteil an Gymnasien.

- Während der Anteil in den Grundschulen im landesweiten Durchschnitt bei rund 10 Prozent lag, betrug er in den Schulamtsbezirken Frankfurt, Offenbach und Groß-Gerau/Main-Taunus rund 20 Prozent.

Der hohe Anteil von Lehrkräften ohne Lehramtsabschluss korreliert eindeutig mit der Sozialstruktur der Region: Der Anteil der Grundschüler*innen mit Migrationshintergrund war im Schuljahr 2016/2017 nirgends höher als in den Schulamtsbereichen Frankfurt (59,4\%) und Groß-Gerau (50,2\%) bzw. in der Stadt Offenbach (71,3\%). Der gleiche Befund ergibt sich bei der Betrachtung der Kinder von sechs bis neun Jahren mit Hilfe zum Lebensunterhalt (SGB II) (Weishaupt, 2018).

Auch dieser Sachverhalt ist dem Lehrkräftemangel und den Verschiebungen auf dem Arbeitsmarkt geschuldet. Inzwischen können sich nicht nur die Lehrkräfte mit gymnasialem Lehramt und einem Mangelfach, sondern auch die Lehrkräfte mit dem Lehramt Grundschule die Schule „aussuchen“, so dass Unterrichtsbedarf

2 Kleine Anfrage des Abgeordneten Christoph Degen (SPD); DS 20/632.

3 Kleine Anfrage des Abgeordneten Christoph Degen (SPD), betreffend Lehrkräftemangel in Hessen; DS 19/4523. 
an den Schulen in einem „weniger begehrten“ Umfeld $\mathrm{zu}$ einem erhöhten Anteil von Seiteneinsteiger*innen führt mit der Folge, dass der Lehrkräftemangel nach der Einschätzung von Klaus Klemm „die soziale Schieflage an den Grundschulen weiter verstärken“" wird:

„Diese Dynamik gewinnt noch zusätzlich an Brisanz durch den Fakt, dass sich hochqualifizierte Lehrkräfte lieber an Schulen in einem gut situierten Umfeld bewerben statt an Brennpunktschulen, obwohl sie dort dringend benötigt werden, um die schwachen Schüler zu fördern. Umgekehrt unterrichten an Problemschulen schon jetzt überproportional viele Seiteneinsteiger, die keine grundständige pädagogische Ausbildung haben." (Klemm, im Interview mit Schubert, 2019)

\section{Quereinsteigerprogramme in Hessen}

Mit einer bundesweiten Kampagne „Hessen sucht Lehrkräfte“ reagierte Hessen im Jahr 2008 erstmals auf den sich abzeichnenden Lehrkräftemangel, der sich zunächst in Problemen bei der Abdeckung von Mangelfächern vor allem im Bereich der Gymnasien und beruflichen Schulen dokumentierte. Nach längeren, auch gerichtlichen Auseinandersetzungen folgte im Juli 2009 eine Änderung des Hessischen Lehrerbildungsgesetzes (HLbG) mit der gesetzlichen Ermächtigung zum Erlass einer „Verordnung über das besondere berufsbegleitende Verfahren zum Erwerb einer einem Lehramt gleichgestellten Qualifikation“, die in Hessen auch offiziell den Namen "Quereinsteiger-Verordnung" trägt. ${ }^{4}$

Die Bewerber*innen mussten „über einen universitären Abschluss“ verfügen, „aus dem in der Regel zwei verschiedene Unterrichtsfächer oder ein Unterrichtsfach und eine Fachrichtung oder zwei Fachrichtungen aus dem jeweils für das gleichzusetzende Lehramt gültigen Fächerkanon ableitbar sind“, sowie „eine mindestens fünfjährige Berufserfahrung im studierten Berufsfeld, das sich auf den schulischen Mangel beziehen muss, “ nachweisen. Die Auswahl erfolgte über eine schulbezogene Stellenausschreibung; die Ausbildung wurde berufsbegleitend im Rahmen eines befristeten Arbeitsverhältnisses durchgeführt und endete mit einer Prüfung. Die Gleichstellung mit dem Lehramt ermöglichte eine Entfristung, eine Gleichstellung der Besoldung und auch eine Verbeamtung. Ohne weitere Begründung und ohne jede Evaluation der eingeleiteten Qualifizierungsmaßnahmen wurde das Programm durch einen Erlass von Kultusministerin Henzler (FDP) vom 01.12.2011 abgebrochen. Bereits eingeleitete Maßnahmen wurden noch zu Ende geführt.

Hessen kehrte damit zu der Praxis der Einstellung von „Vertretungslehrkräften“ zurück, die ohne eine ernsthafte Prüfung ihrer individuellen Voraussetzungen und

4 Amtsblatt des Hessischen Kultusministerium 8/2009. 
Qualifikationen und ohne jedes Fortbildungsangebot vom ersten Tag an mit voller Stundenzahl im Unterricht eingesetzt werden können.

Die Regelungen der Verordnung wurden zwar weitgehend wortgleich in die Durchführungsverordnung zum Hessischen Lehrerbildungsgesetz (HLbG-DV) übernommen, doch werden sie nur in wenigen Fällen, vor allem an beruflichen Schulen, angewendet. Strukturierte, systematische Maßnahmen zur Personalgewinnung und Personalentwicklung unterblieben, so dass sich Hessens Schulen bei der Bewältigung des Lehrkräftemangels weiter von Schuljahr zu Schuljahr hangeln müssen.

\section{Reaktionen auf den verschärften Lehrkräftemangel ab 2016}

Auf die Verschärfung des Lehrkräftemangels in den Jahren 2016 und 2017 insbesondere an den Grundschulen reagierte das HKM mit der vermehrten Einstellung von befristet beschäftigten Vertretungslehrkräften. Außerdem sollen folgende Maßnahmen kurz- und mittelfristig eine Entspannung bringen:

- Bewerber*innen für den Vorbereitungsdienst, die das Erste Staatsexamen für das Lehramt an Gymnasien absolviert haben, erhalten die Möglichkeit für einen Quereinstieg in das Grundschulreferendariat.

- Lehrkräfte mit dem Lehramt an Gymnasien bzw. an Haupt- und Realschulen, die nach dem Zweiten Staatsexamen keine Einstellung gefunden haben, können sich auf eine dreijährige berufsbegleitende Weiterbildung für das Lehramt an Grundschulen bewerben. Sie verpflichten sich in dem Arbeitsvertrag, nach dem Bestehen der Zusatzprüfung und der Verbeamtung im Grundschullehramt mindestens drei weitere Jahre als Grundschullehrkraft tätig zu sein. Die Besoldung erfolgt nach A 12. Danach ist eine Bewerbung auf eine Stelle möglich, die der „zuvor erworbenen Lehramtsbefähigung“ entspricht.

- Diese mit einer umfassenden Weiterbildung und einer deutlichen Orientierung auf einen Verbleib in der Grundschule verbundene Maßnahme wird aktuell im HKM durch ein neues Programm konterkariert, wonach solche Lehrkräfte mit gymnasialem Lehramtsabschluss bevorzugt eingestellt werden sollen, die im Rahmen ihrer Bewerbung auf der Rangliste ihre Bereitschaft erklären, nach der Einstellung an einem Gymnasium für eine begrenzte Zeit mit einem Teil ihrer Pflichtstundenzahl an einer Grundschule $\mathrm{zu}$ unterrichten. Einstellung und Verbeamtung auf Lebenszeit erfolgen jedoch am Gymnasium. Damit erhalten diese Lehrkräfte anders als die Kolleg*innen, die sich auf die Weiterbildung einlassen, auch die gymnasiale Besoldung.

Aktuell arbeitet das HKM außerdem an einer Änderung der Durchführungsverordnung zum HLbG, um die Eingangsvoraussetzungen für einen Quereinstieg ab- 
zusenken. Danach soll der Quereinstieg zukünftig auch für Personen mit einem Bachelorabschluss möglich sein, aus dem „mindestens ein Unterrichtsfach für das Grundschullehramt ableitbar ist“. Die Bedingungen für die professionelle Qualifizierung für den Lehrberuf und für die Nachqualifizierung für das zweite Fach sind noch nicht bekannt. Dasselbe gilt für die Frage, ob am Ende der Maßnahme eine von allen Bundesländern nach den Kriterien der KMK für die „Gestaltung von Sondermaßnahmen zur Gewinnung von Lehrkräften “5 anerkannte Gleichstellung mit einem Lehramt erfolgt, die auch den Zugang zu einer gleichwertigen Eingruppierung bzw. zu einer Verbeamtung eröffnet.

\section{Forderung der Lehrerinnenverbände für den Quereinstieg in Hessen}

Der Lehrkräftemangel wird auch in Hessen weiter konsequente Maßnahmen zur Gewinnung von Lehrkräften erforderlich machen. Sowohl die für Hessen vorgelegte Expertise von Dieter Dohmen und Maren Thomsen (2018) als auch die Übertragung der jüngsten Prognosen von Klaus Klemm und Dirk Zorn (2019) auf Hessen (EickerWolf, 2019) zeigen einen wachsenden Bedarf an gut ausgebildeten Lehrkräften, wobei der Mangel aus den Grundschulen in die weiterführenden Schulen aufwachsen wird.

Der Landesverband Hessen der Gewerkschaft Erziehung und Wissenschaft (GEW) hält es deshalb für erforderlich, über die Maßnahmen zur Ausweitung der Zahl der Studienplätze in den Lehramtsstudiengängen an hessischen Universitäten und die entsprechende Erhöhung der Zahl der Stellen im Vorbereitungsdienst hinaus auch Seiteneinsteiger*innen für den Lehrberuf zu motivieren und berufsbegleitend umfassend zu qualifizieren. ${ }^{6}$ Dabei hält die GEW das von Klaus-Jürgen Tillmann (2020) vorgestellte Qualifizierungsmodell im Grundsatz für geeignet, den Quereinstieg zu gestalten (vgl. auch den Beitrag von Tillmann in diesem Heft).

Ein Konzept für eine umfassende berufsbegleitende Qualifizierung muss aus Sicht der GEW folgende Kriterien berücksichtigen:

- Sicherung der Unterrichtsqualität;

- Einstiegsqualifizierung vor Beginn des Unterrichtseinsatzes;

- ausreichende Entlastung der Kolleg*innen bei der Unterrichtsverpflichtung zur Erfüllung der Qualifizierungsauflagen;

- ausreichende Entlastung der Kolleg*innen, die sich in den Praxisphasen als Mentor*innen engagieren;

- Sicherstellung einer attraktiven Bezahlung in allen Phasen der Qualifizierung;

5 Beschluss der KMK von 05.12.2013.

6 Beschluss des GEW-Landesvorstands 216neu/2018-2020. 
- Ermöglichung eines Abschlusses, der mit dem Erwerb eines Lehramts oder einer Gleichstellung verbunden ist.

Für die bisher im Rahmen einer „Unterrichtserlaubnis“ unbefristet beschäftigten Bestandslehrkräfte fordert die GEW ein umfassendes Qualifizierungsprogramm, das diesen schon lange eingesetzten Kolleg^innen eine dauerhafte Berufsperspektive und eine der Tätigkeit angemessene Bezahlung ermöglicht.

\section{Ausblick}

Weil allein in Hessen mehr als 5.000 Lehrkräfte in der unterprivilegierten Position einer (meist zeitlich befristeten) Vertretungslehrkraft arbeiten, liegt es nahe, nach der Situation in den anderen Bundesländern und damit auch nach einer Gesamtzahl solcher "Vertretungslehrkräfte“ in der Bundesrepublik zu fragen. Weil die meisten Kultusministerien hierzu aber keine Zahlen veröffentlichen, lässt sich eine datengestützte Schätzung kaum vornehmen. Es kommt hinzu, dass diese Gruppe der Lehrkräfte bisher auch nicht in den Blick der erziehungswissenschaftlichen Forschung geraten ist. So gesehen führt sie bislang eher ein Schattendasein. Dieser Beitrag will einen Anstoß geben, die bildungspolitische wie die erziehungswissenschaftliche Auseinandersetzung mit dieser problematischen Form der Lehrkräftebeschäftigung deutlich zu befördern.

\section{Literatur und Internetquellen}

Dohmen, D., \& Thomsen, M. (2018). Prognose der Schülerzahl und des Lehrkräftebedarfs an allgemeinbildenden Schulen in Hessen bis 2030. Endbericht einer Studie für die Fraktion Die Linke im Hessischen Landtag. Berlin: Forschungsinstitut für Bildungsund Sozialökonomie. Zugriff am 14.10.2020. Verfügbar unter: https://www.fibs.eu/file admin/user_upload/Literatur/FiBS_LinkeHE_LK-Bedarf_180806.pdf.

Eicker-Wolf, K. (2019). Lehrkräftemangel an Grundschulen. HLZ - Zeitschrift der GEW Hessen für Erziehung, Bildung, Forschung, 72 (9), 9.

Klemm, K., \& Zorn, D. (2019). Steigende Schülerzahlen im Primarbereich: Lehrkräftemangel deutlich stärker als von der KMK erwartet. Gütersloh: Bertelsmann Stiftung. Zugriff am 10.08.2020. Verfügbar unter: https://www.bertelsmann-stiftung.de/de/publikationen/ publikation/did/steigende-schuelerzahlen-im-primarbereich-lehrkraeftemangel-deut lich-staerker-al s-von-der-kmk-erwartet/.

KMK (Sekretariat der Ständigen Konferenz der Kultusminister der Länder in der Bundesrepublik Deutschland). (2019). Einstellung von Lehrkräften 2018. Statistische Veröffentlichungen der Kultusministerkonferenz, Dokumentation Nr. 218. Zugriff am 19.08.2020. Verfügbar unter: https://www.kmk.org/fileadmin/Dateien/pdf/Statistik/ Dokumentationen/Dok_218_EvL_2018.pdf.

KMK (Sekretariat der Ständigen Konferenz der Kultusminister der Länder in der Bundesrepublik Deutschland). (2020). Einstellung von Lehrkräften 2019. Tabellenauszug. 
Zugriff am 19.08.2020. Verfügbar unter: https://www.kmk.org/fileadmin/Dateien/pdf/ Statistik/Dokumentationen/Tabellenauszug_EvL_2019.pdf.

Schubert, F. (2019, 10. Oktober). Lehrermangel: Kinder werden um Bildung betrogen. Interview mit K. Klemm. Frankfurter Rundschau. Zugriff am 19.08.2020. Verfügbar unter: https://www.fr.de/wissen/lehrermangel-kinder-werden-bildung-betrogen-12986461. html.

Tillmann, K. J. (2019). Von einer Notmaßnahme zu einem dauerhaften Konzept? Der Seiteneinstieg in den Lehrerberuf. Pädagogik, (6), 11-14.

Tillmann, K. J. (2020). Zwei Wege zum Lehrerberuf. Seiteneinstieg und pädagogische Professionalität. HLZ - Zeitschrift der GEW Hessen für Erziehung, Bildung, Forschung, 73 (1-2), 12-13.

Weishaupt, H. (2018). Kinderarmut und Migration. Personalausstattung der Schulen und schulische Lernbedingungen. HLZ - Zeitschrift der GEW Hessen für Erziehung, Bildung, Forschung, 71 (3), 24-25.

Harald Freiling, geb. 1951, verantwortlicher Redakteur der HLZ, der Mitgliederzeitschrift der Gewerkschaft Erziehung und Wissenschaft (GEW) Hessen, und Mitglied des geschäftsführenden GEW-Landesvorstands.

E-Mail: freiling.hlz@t-online.de

Korrespondenzadresse: Klingenbergerstr. 13, 60599 Frankfurt a.M. 